\title{
Corrigendum
}

\section{Acute Stress Responsiveness of the Neurotrophin BDNF in the Rat Hippocampus is Modulated by Chronic Treatment with the Antidepressant Duloxetine}

Raffaella Molteni, Francesca Calabrese, Annamaria Cattaneo, Michele Mancini, Massimo Gennarelli, Giorgio Racagni and Marco A Riva

Neuropsychopharmacology (2009) 34, 2196; doi:10.1038/npp.2009.44

Correction to: Neuropsychopharmacology (2008) 34, 1-10.

doi:10.1038/npp.2008.208

In this article, the author has identified an error on page 5, in "panel e" of Figure 2; the label should be "Exon VI", not "Exon IV". 